\title{
Myelodysplastic Syndrome
}

National Cancer Institute

\section{Source}

National Cancer Institute. Myelodysplastic Syndrome. NCI Thesaurus. Code C3247.

A clonal hematopoietic disorder characterized by dysplasia and ineffective hematopoiesis in one or more of the hematopoietic cell lines. The dysplasia may be accompanied by an increase in myeloblasts, but the number is less than $20 \%$, which, according to the WHO guidelines, is the requisite threshold for the diagnosis of acute myeloid leukemia. It may occur de novo or as a result of exposure to alkylating agents and/or radiotherapy. (WHO, 2001) 